\title{
Análise da deformação na prótese femoral não cimentada do quadril pelo método de elementos finitos - Influência da variabilidade do posicionamento angular do implante*
}

\section{Strain Analysis in Cementless Hip Femoral Prosthesis using the Finite Element Method - Influence of the Variability of the Angular Positioning of the Implant}

Felipe Alberto Degasperi ${ }^{1 \oplus}$ Bruno Francesco Scatigna ${ }^{2}$ Guilherme Guadagnini Falótico $^{2}{ }^{\circ}$ Valéria Romero ${ }^{3(0)}$ Ricardo Basile ${ }^{4(1)}$ Edmilson Takehiro Takata2 ${ }^{(1)}$

${ }^{1}$ Instituto ESSS de Educação, Pesquisa e Desenvolvimento, Piracicaba, SP, Brasil

${ }^{2}$ Grupo de patologias do quadril adulto, Escola Paulista de Medicina, Universidade Federal de São Paulo, São Paulo, SP, Brasil

3 Universidade Estadual de Campinas, Campinas, SP, Brasil

${ }^{4}$ Escola Paulista de Medicina, Universidade Federal de São Paulo, São Paulo, SP, Brasil

Endereço para correspondência Bruno Francesco Scatigna, MD, Universidade Federal de São Paulo, Rua Napoleão de Barros, 715, $1^{\circ}$ andar; São Paulo, SP, 04024-002, Brasil (e-mail: bruno.scatigna@unifesp.br).

Rev Bras Ortop 2022;57(6):968-974.

\section{Resumo}

\section{Palavras-chave}

- artroplastia de quadril

- prótese de quadril

- análise de elementos finitos

- análise de falha de equipamento
Objetivo Avaliar a influência da variação do posicionamento da prótese femoral do quadril na tensão e na deformação produzidas neste implante.

Métodos Utilizou-se a análise de tensão e de deformação da prótese femoral (Taper, Víncula, Rio Claro, SP, Brasil) pelo método de elementos finitos (MEF) de acordo com a norma ISO 7206-6 Implants for surgery - Partial and total hip joint prostheses-Part 6: Endurance properties testing and performance requirements of neck region of stemmed femoral components. A análise propôs uma ramificação do ensaio físico, com variação da angulação de $+/-5^{\circ}$ sobre a proposta normativa das variáveis $\alpha$ e $\beta$.

Resultados Ao comparar com a deformação controle, houve significância estatística com a angulação isolada de $+\mid-5^{\circ}$ do ângulo $\alpha$, bem como com a associação de $+\mid-5^{\circ}$ nas angulações $\alpha$ e $\beta$ ( $p=0,027$ e 0,021 , respectivamente). Já com a variação apenas do ângulo $\beta$, não houve variação significativa na deformação da prótese $(p=0,128)$. A posição da haste com maior deformação no implante foi com $\alpha=5^{\circ}$ e $\beta=14^{\circ}(p=0,032)$.

\footnotetext{
Trabalho desenvolvido na Escola Paulista de Medicina, Universidade Federal de São Paulo, São Paulo, SP, Brasil
}

recebido

12 de Janeiro de 2021

aceito

12 de Abril de 2021

Publicado on-line

Dezembro 13, 2021
DOI https://doi.org/ $10.1055 / \mathrm{s}-0041-1735141$ ISSN $0102-3616$
(C) 2021. Sociedade Brasileira de Ortopedia e Traumatologia. All rights reserved.

This is an open access article published by Thieme under the terms of the Creative Commons Attribution-NonDerivative-NonCommercial-License, permitting copying and reproduction so long as the original work is given appropriate credit. Contents may not be used for commercial purposes, or adapted, remixed, transformed or built upon. (https://creativecommons.org/ licenses/by-nc-nd/4.0/)

Thieme Revinter Publicações Ltda., Rua do Matoso 170, Rio de Janeiro, RJ, CEP 20270-135, Brazil 


\section{Abstract}

\section{Keywords}

- arthroplasty, replacement, hip

- hip prosthesis

- finite element analysis

- equipment failure analysis
Conclusão A variabilidade de posicionamento da haste femoral protética de $+1-5^{\circ}$ no plano coronal e/ou a associação da angulação de $+/-5^{\circ}$ nos planos coronal e sagital interferiu de forma significativa na deformação do implante.

Objective The present study aims to evaluate the influence of different positioning of the hip femoral prosthesis on the stress and strain over this implant.

Methods A femoral prosthesis (Taper-Víncula, Rio Claro, SP, Brazil) was submitted to a stress and strain analysis using the finite element method (FEM) according to the International Organization for Standardization (ISO) 7206-6 Implants for surgery - Partial and total hip joint prostheses - Part 6: Endurance properties testing and performance requirements of neck region of stemmed femoral components standard. The analysis proposed a branch of the physical test with $\mathrm{a}+1-5^{\circ}$ angle variation on the standard proposed for $\alpha$ and $\beta$ variables.

Results The isolated $+1-5^{\circ}$ variation on the $\alpha$ angle, as well as the association of $+/-5^{\circ}$ variation on the $\alpha$ and $\beta$ angles, presented significant statistical differences compared with the control strain ( $p=0.027$ and 0.021 , respectively). Variation on angle $\beta$ alone did not result in a significant change in the strain of the prosthesis $(p=0.128)$. The stem positioning with greatest implant strain was $\alpha=5^{\circ}$ and $\beta=14^{\circ}$ $(p=0.032)$.

Conclusion A variation on the positioning of the prosthetic femoral stem by $+/-5^{\circ}$ in the coronal plane and/or the association of $\mathrm{a}+1-5^{\circ}$ angle in coronal and sagittal planes significantly influenced implant strain.

\section{Introdução}

A artroplastia total de quadril (ATQ) é uma das cirurgias mais bem-sucedidas da atualidade, com excelentes resultados a longo prazo. No entanto, o sucesso do procedimento depende de diversos fatores - indicação cirúrgica correta, planejamento adequado e técnica operatória efetiva. ${ }^{1}$

As cargas cíclicas impostas sobre o quadril durante as mais diversas atividades são extremamente elevadas. ${ }^{2} \mathrm{~A}$ articulação protética deve estar preparada para suportar tais cargas de tensão resistindo à deformação imposta. Cabe ao cirurgião ortopédico reconstruir da forma mais adequada possível a biomecânica do quadril na artroplastia, restaurando momento da força de força muscular garantindo sobrevida a longo prazo do implante. Neste cenário, o correto posicionamento dos componentes protéticos é parte fundamental na transferência harmônica das cargas no quadril e no bom funcionamento mecânico da articulação. ${ }^{3}$

No ambiente experimental, a fim de determinar as propriedades de resistência à fadiga de uma haste femoral protética, são realizados ensaios físicos pré-clínicos, tais como os preconizados pelas normativas International Organization for Standardization (ISO) 7206-4 e ISO 7206-6 (-Figura 1). ${ }^{4,5}$ A resistência do material é avaliada pelo comportamento da curva tensão-deformação, sendo que, nos ensaios convencionais aumenta-se a carga até a ruptura do material. No método de elementos finitos (MEF), uma simulação computacional do comportamento do implante frente às cargas cíclicas é realizada baseada em testes físicos simplificados e nas propriedades físico-químicas previa- mente conhecidas do material, diminuindo o tempo de execução e o custo em relação às simulações biomecânicas tradicionais. ${ }^{6}$

Os ensaios biomecânicos habituais das hastes femorais preconizam o aumento progressivo das cargas cíclicas com o componente femoral em posição angular fixa. ${ }^{7,8}$ Assim, os testes desprezam o comportamento das hastes femorais implantadas em varo ou valgo, antecurvo ou recurvo, situações frequentemente observadas na prática clínica e que podem influenciar de forma determinante na sobrevida do implante a longo prazo. Assim, o objetivo do presente estudo é avaliar, pelo MEF, a influência da variabilidade do posicionamento angular de uma haste femoral protética sobre a tensão e a deformação no implante.

\section{Material e métodos}

Inicialmente, foi realizado ensaio físico da haste femoral protética (Taper - Víncula, Rio Claro, SP, Brazil) (-Figura 2), em posição angular fixa conforme as normas ISO 7206-4 (-Figura 1) e ISO 7206-6., ${ }^{4,5}$ Esta haste é de desenho anatômico, em tripla-cunha em titânio, de método de fixação não cimentado e porosidades proximais para osteointegração.

Baseado nos dados do ensaio biomecânico inicial e nas propriedades físico-químicas da prótese, partiu-se para a o desenvolvimento do presente estudo. Para a análise, foi utilizada a plataforma Ansys Workbench 19.1, um portal de engenharia virtual online (ANSYS, Canonsburg, PA, EUA), utilizando o recurso "solver static strutural" da plataforma associado paralelamente à parametrização dos componentes 


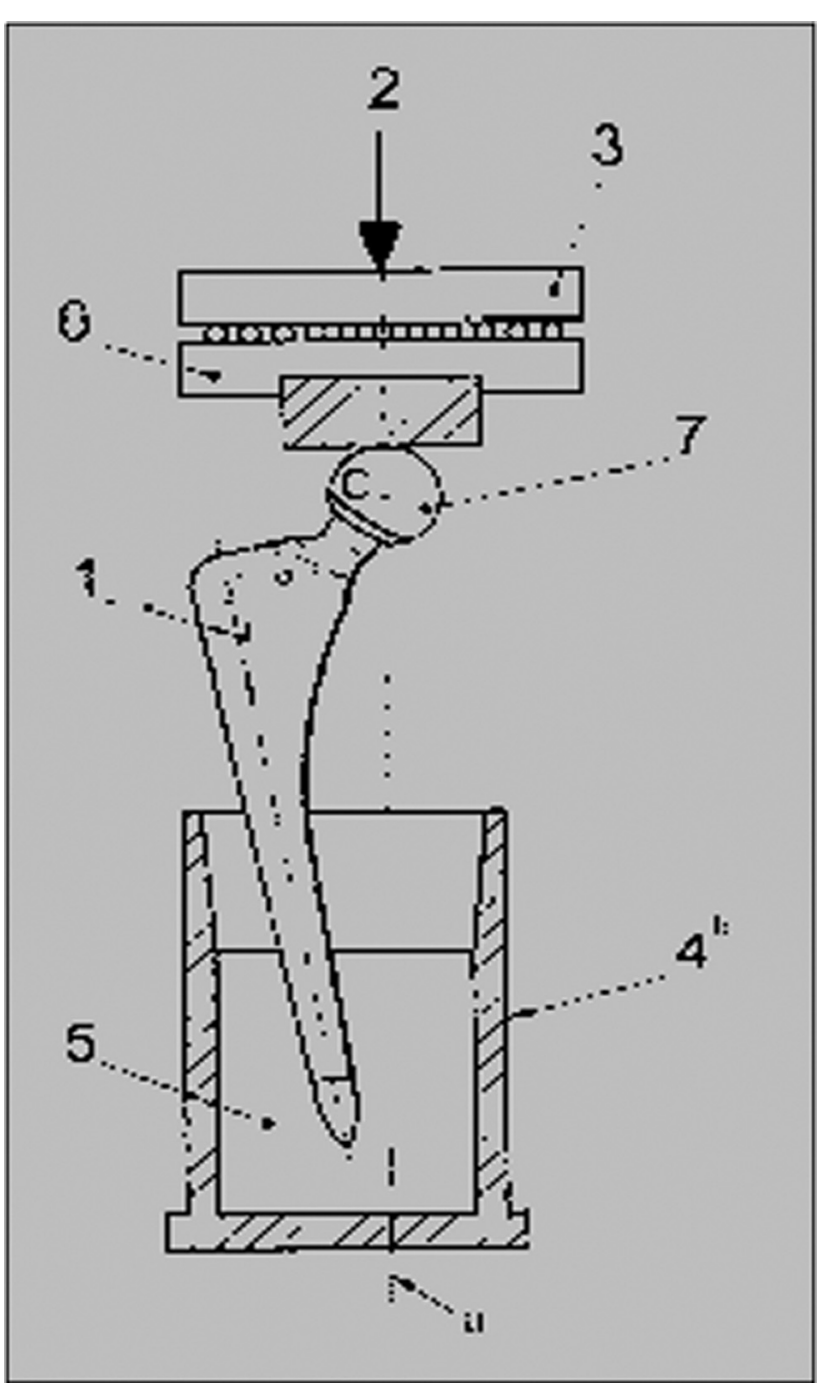

Fig. 1 Ensaio mecânico ISO 7206-4. Fonte: Imagem de ISO 7206-4, 2010.

do vetor da carga. Esta análise adicional tem o objetivo de propor uma ramificação do ensaio físico com variação de $+/-5^{\circ}$ nos planos coronal e sagital, em relação ao preconizado no documento normativo da ISO.

As características do material inseridas na plataforma foram obtidas na norma ASTM F136 - Standard Specification for Wrought Titanium-6Aluminum-4Vanadium ELI (Extra Low Interstitial) Alloy for Surgical Implant Applications (ASTM F136, 2013) ${ }^{9}$ (- Tabela 1). O módulo de elasticidade deste material é aproximadamente $110 \mathrm{Gpa}$; no entanto, a resistência a cisalhamento deste material é relativamente baixa.

Tabela 1 Propriedades do material

\begin{tabular}{|l|l|}
\hline Propriedades & Forjado (F136) \\
\hline Tensão de ruptura [MPa] & 860 \\
\hline Tensão de escoamento [MPa] & 795 \\
\hline Alongamento [\%] & 10 \\
\hline Redução de área [\%] & 20 \\
\hline
\end{tabular}

Fonte: Tabela adaptada de Park \& Lakes, 2007.

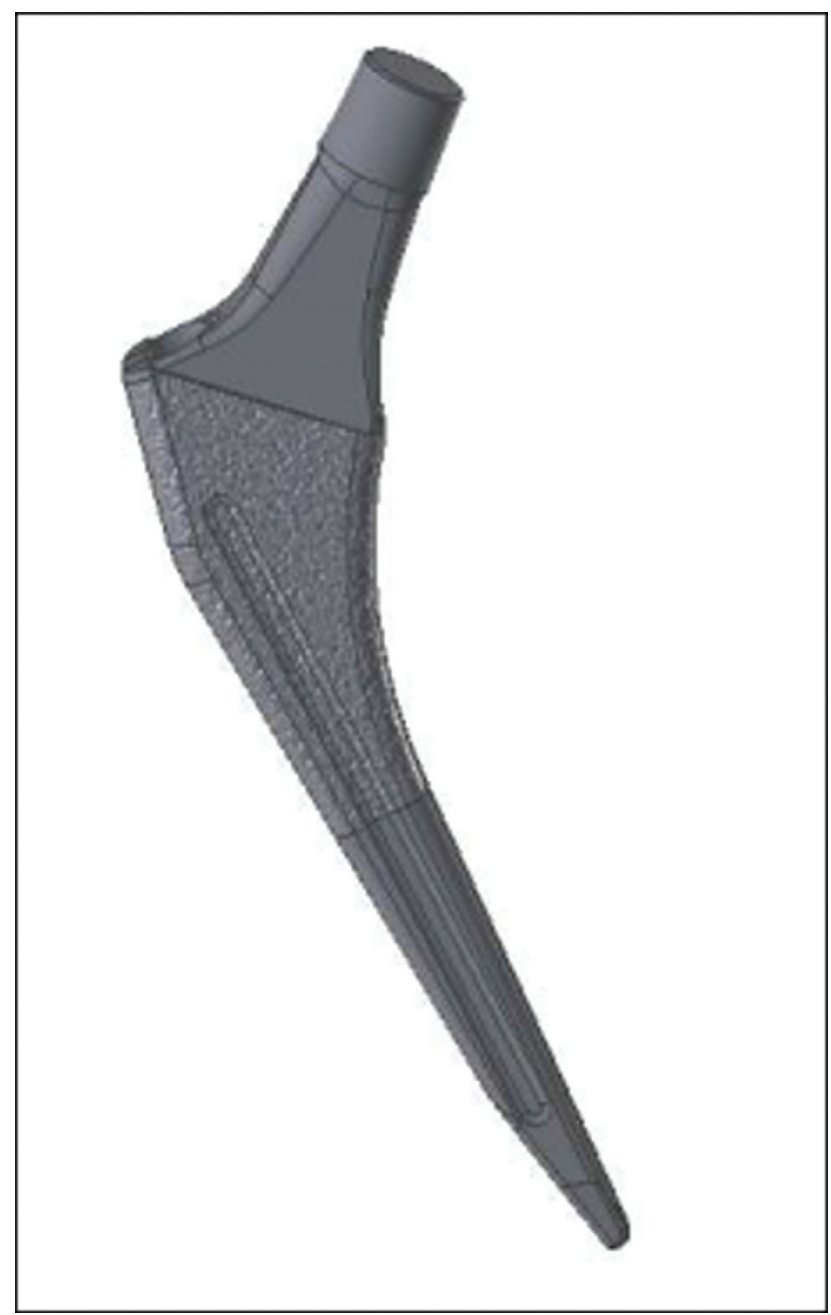

Fig. 2 Representação da geometria. Fonte: Acervo do autor.

As propriedades físicas da liga de titânio são: tensão de ruptura de 780 a $1.050 \mathrm{GPa}$, densidade de $4,4 \mathrm{~g} / \mathrm{cm}^{3}$ e coeficiente de Poisson de $0,34 .^{10}$ Adotou-se, para efeito de análise computacional, o comportamento do material sob cargas como perfeitamente plástico (—Figura 3 ).

\section{Condições de contorno (carregamento e restrições de movimento)}

O teste foi realizado seguindo a proposta descrita na norma ISO 7206-6, ${ }^{5}$ porém com restrição de movimento na prótese durante a aplicação de cargas, condição conhecida como "bonded" (-Figura 4). Com a intenção de eliminar concentradores de tensão na região de interesse principal do estudo (transição colo-corpo), o engastamento foi realizado $10 \mathrm{~mm}$ abaixo da proposta descrita pela norma técnica. Para o carregamento, foi aplicado um vetor no cone, onde habitualmente é realizado o acoplamento com a cabeça femoral, simulando o centro de rotação do sistema (-Figura 4).

A variação dos componentes da carga foi calculada através da decomposição do vetor de carga (-Figura 5 ). 0 posicionamento padrão da haste femoral conforme norma técnica citada acima foi de ângulos $\alpha=10^{\circ}$ e $\beta=9^{\circ}$ e a força aplicada de $5.340 \mathrm{~N}$. Testes computacionais subsequentes foram realizados com aplicação da mesma força nas diferentes 


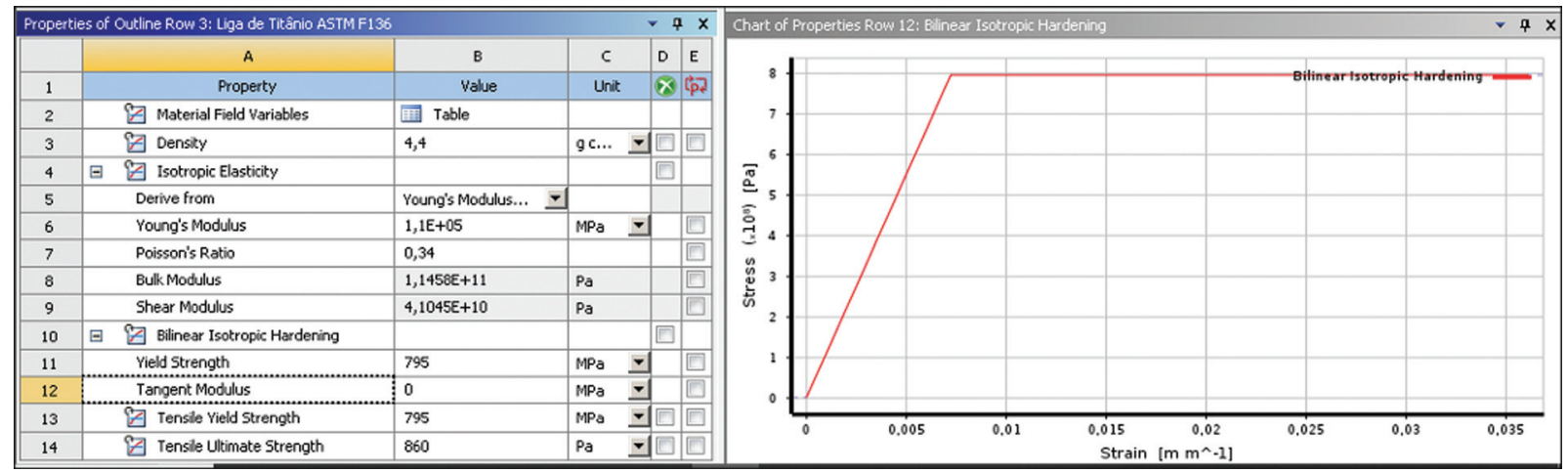

Fig. 3 Propriedades do material. Fonte: Acervo do autor.

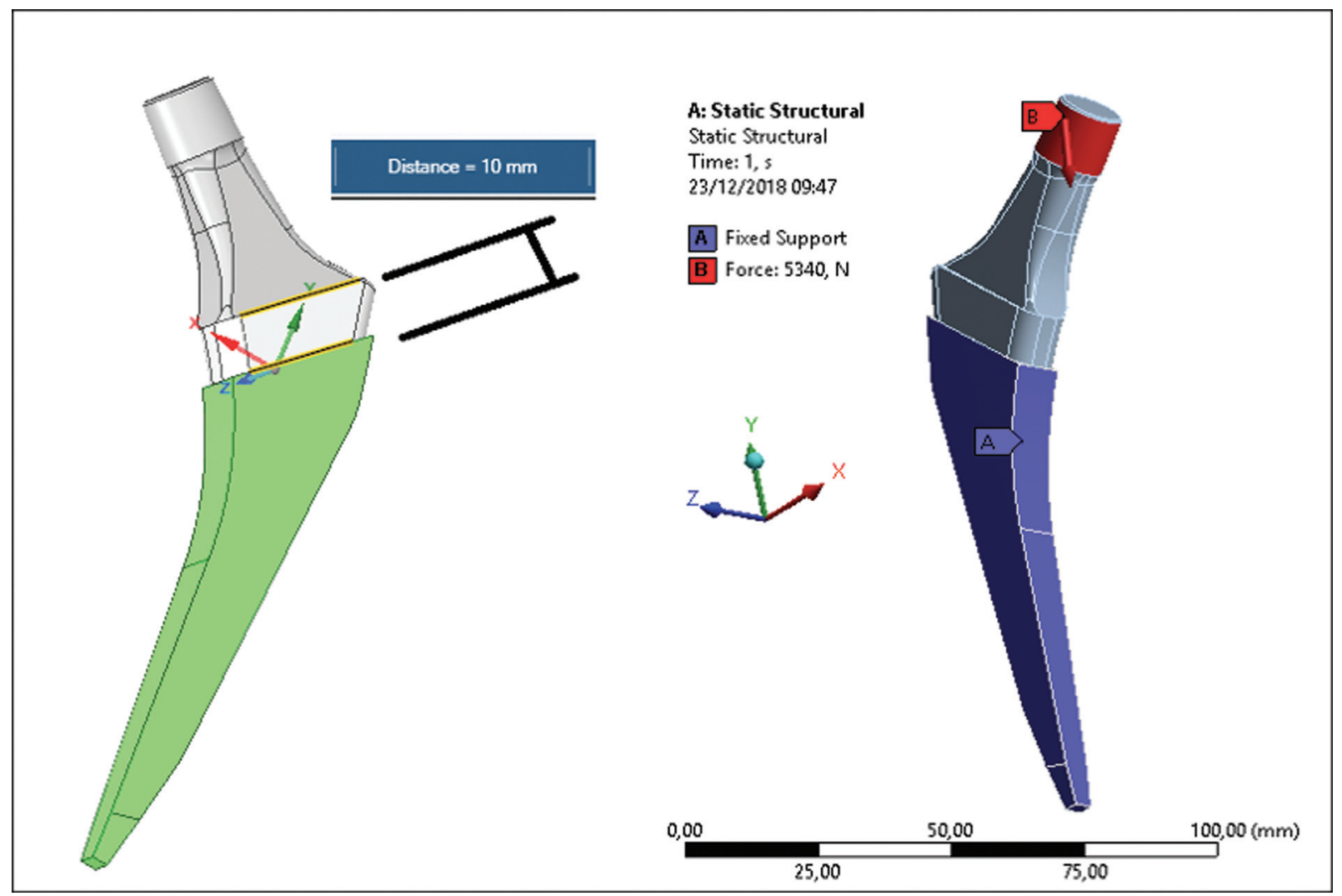

Fig. 4 Condições de contorno. Fonte: Acervo do autor.

combinações de ângulos $\alpha$ e $\beta+/-5^{\circ}$, conforme demonstrado na - Tabela 2.

\section{Modelo de elementos finitos (malha)}

Para a construção da malha a ser interpretada pelo sistema, utilizamos o elemento sólido tetraédrico parabólico com tamanho médio de $3 \mathrm{~mm}$ preenchendo a região do corpo da prótese. Para o bloco cone-colo, um elemento hexaédrico dominante com tamanho médio de $2 \mathrm{~mm}$ (-Figura 6).

Para a escolha da área da haste para o teste de tensão $\mathrm{x}$ deformação, avaliamos a tensão equivalente em 6 regiões distintas da prótese, escolhida então a área de maior tensão equivalente (transição colo-corpo - ponto de acoplamento do introdutor da haste), conforme demonstrado na -Tabela 3 e na - Figura 7.

\section{Análise estatística}

Análise estatística realizada com softwares Excel Office 2010 (Microsoft Corp., Redmond, WA, EUA) e SPSS V20 (IBM Corp., Armonk, NY, EUA). As comparações entre as variáveis foram realizadas por meio do teste te Student, após verificação da normalidade e da variância dos dados. $\mathrm{O}$ valor de $p$ utilizado como significativo foi de 0,05 e o intervalo de confiança de $95 \%$.

\section{Resultados}

A - Tabela 4 descreve resultados observados de tensão e deformação na região de transição colo corpo da haste nos diferentes posicionamentos.

A análise estatística foi separada em 3 cenários: 
972 Análise da deformação na prótese femoral não cimentada do quadril Degasperi et al.

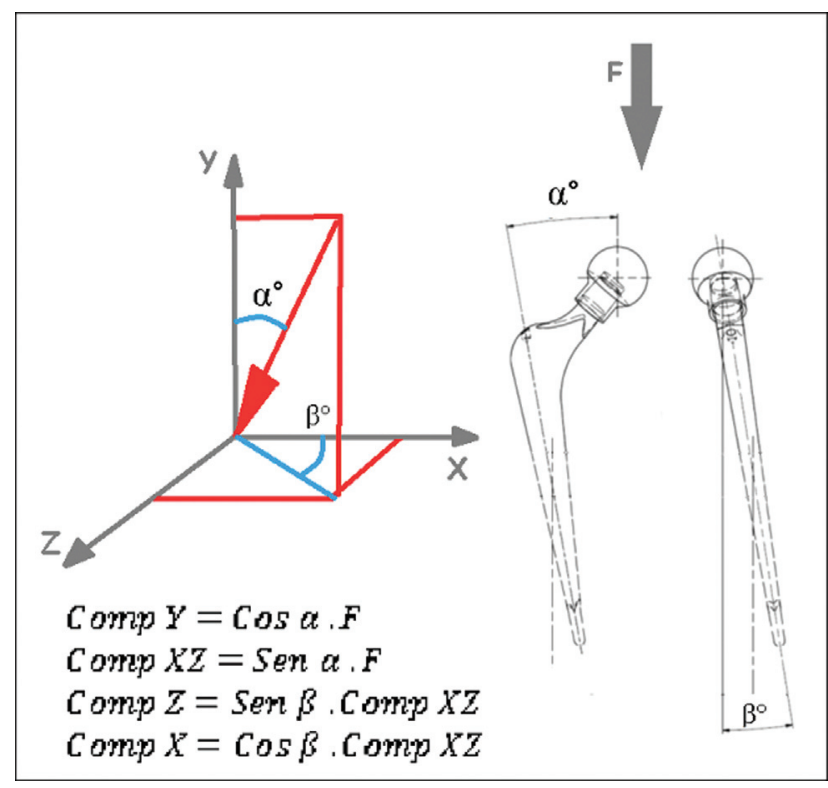

Fig. 5 Componentes da força. Fonte: Acervo do autor.

1) comparação da deformação do teste na posição padrão $\left(\alpha=10^{\circ}\right.$ e $\left.\beta=9^{\circ}\right)$ variando apenas o ângulo $\alpha\left(5^{\circ}\right.$ e $\left.15^{\circ}\right)$

2) comparação da deformação do teste na posição padrão $\left(\alpha=10^{\circ}\right.$ e $\left.\beta=9^{\circ}\right)$ variando apenas o ângulo $\beta$ $\left(4^{\circ}\right.$ e $\left.14^{\circ}\right)$
Tabela 2 Variação da angulação na carga

\begin{tabular}{|l|l|l|l|l|}
\hline$\alpha^{\circ}$ & $\beta^{\circ}$ & Comp X [N] & Comp Y [N] & Comp Z [N] \\
\hline 10 & 9 & 915,86 & $5.258,87$ & 145,06 \\
\hline 10 & 4 & 925,02 & $5.258,87$ & 64,68 \\
\hline 10 & 14 & 899,74 & $5.258,87$ & 224,33 \\
\hline 5 & 9 & 459,68 & $5.319,68$ & 72,81 \\
\hline 5 & 4 & 464,28 & $5.319,68$ & 32,47 \\
\hline 5 & 14 & 451,59 & $5.319,68$ & 112,59 \\
\hline 15 & 9 & $1.365,08$ & $5.158,04$ & 216,21 \\
\hline 15 & 4 & $1.378,73$ & $5.158,04$ & 96,41 \\
\hline 15 & 14 & $1.341,04$ & $5.158,04$ & 334,36 \\
\hline
\end{tabular}

3) comparação da deformação do teste na posição padrão variando os ângulos $\alpha$ e $\beta$ ( $\alpha$ de $5^{\circ}$ e $15^{\circ}$ e $\beta$ de $4^{\circ}$ e $14^{\circ}$ )

Nos cenários 1 e 3 , houve significância estatística $(p=0,027$ e 0,021 , respectivamente). Já com a variação apenas do ângulo $\beta$, não houve variação significativa na deformação da prótese $(p=0,128)$.

A posição da haste com maior deformação no implante foi $\operatorname{com} \alpha=5^{\circ}$ e $\beta=14^{\circ}(p=0,032)$.

Nenhuma das solicitações mecânicas testadas levaram o material a falha, pois os valores sobre a resposta não ultrapassaram os valores de escoamento do material.

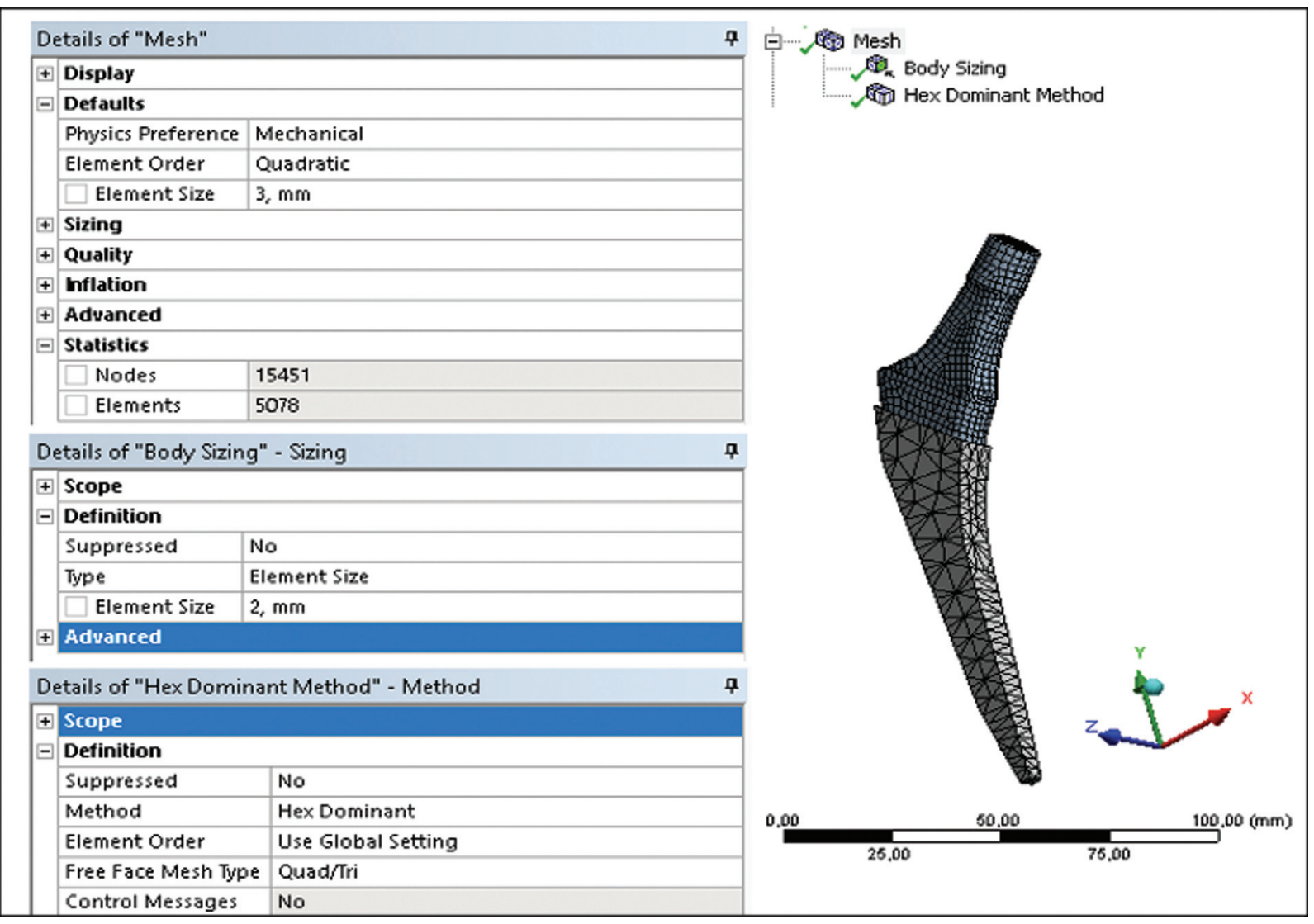

Fig. 6 Modelo de elementos finitos (Malha). Fonte: Acervo do autor. 
Tabela 3 Convergência da malha

\begin{tabular}{|l|l|l|l|}
\hline $\begin{array}{l}\text { Região de } \\
\text { preenchimento } \\
\text { tetraédrico } \\
(\mathrm{mm})\end{array}$ & $\begin{array}{l}\text { Região de } \\
\text { interesse } \\
\text { hexaédrico } \\
(\mathrm{mm})\end{array}$ & $\begin{array}{l}\text { Números } \\
\text { de nós/ } \\
\text { elementos }\end{array}$ & $\begin{array}{l}\text { Tensão } \\
\text { equivalente } \\
\text { (MPa) }\end{array}$ \\
\hline $5 \mathrm{~mm}$ & $4 \mathrm{~mm}$ & $5.155 / 1.818$ & 276,93 \\
\hline $5 \mathrm{~mm}$ & $3 \mathrm{~mm}$ & $7.439 / 2.572$ & 275,56 \\
\hline $5 \mathrm{~mm}$ & $2,5 \mathrm{~mm}$ & $9.880 / 3.318$ & 278 \\
\hline $4 \mathrm{~mm}$ & $2 \mathrm{~mm}$ & $14.913 / 4.816$ & 284,72 \\
\hline $3 \mathrm{~mm}$ & $2 \mathrm{~mm}$ & $15.451 / 5.078$ & 283,92 \\
\hline $2 \mathrm{~mm}$ & $1,5 \mathrm{~mm}$ & $28.908 / 9.508$ & 328,67 \\
\hline
\end{tabular}
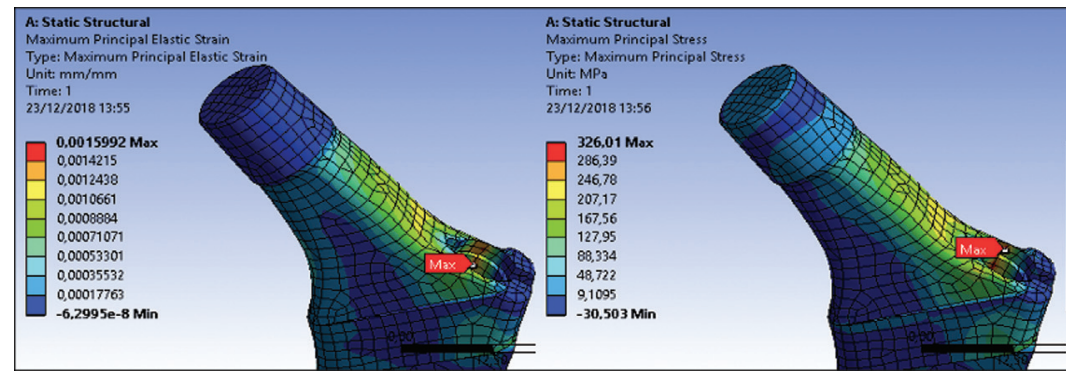

Fig. 7 Região de maior concentração de tensão Fonte: Acervo do autor.

\section{Discussão}

O presente estudo de forma inédita avaliou pelo MEF o efeito da variabilidade de posicionamento do componente femoral sobre a deformação no implante. Os principais achados foram que a variação de $+/-5^{\circ}$ no plano coronal ou a associação de $+1-5^{\circ}$ na angulação no plano coronal e sagital da prótese femoral em teste computacional por MEF apresentaram aumento significativo na deformação do implante.

Goel e Nyman, ${ }^{11}$ em 2016, citaram o potencial do uso do MEF para análise da biomecânica das articulações do corpo humano. Já em 2019, Akrami et al. ${ }^{12}$ descreveram a utilização do MEF para análise da biomecânica do quadril em um estudo baseado nas imagens de ressonância magnética de uma voluntária de 20 anos de idade sem lesões nesta articulação. Esta pesquisa permitiu verificar as propriedades mecânicas da cartilagem, do osso esponjoso e do osso cortical do acetábulo e do fêmur proximal, bem como sua resposta frente a simulações de carga.

Quanto às artroplastias do quadril, Reimeringer e Nuño, ${ }^{13}$ em 2016, estudaram pelo MEF o comportamento do componente femoral não cimentado no canal femoral e demonstraram que o contato total da prótese com o osso hospedeiro não é necessário para a osteointegração; valores entre 25 e $57 \%$ de contato permitiram boa integração óssea. Bitter et al., ${ }^{14}$ em 2017, estudaram o desgaste, pelo MEF, em componentes modulares de ATQ conseguindo prever de forma adequada, em relação a ensaios físicos, a taxa esperada de desgaste anual do sistema protético.

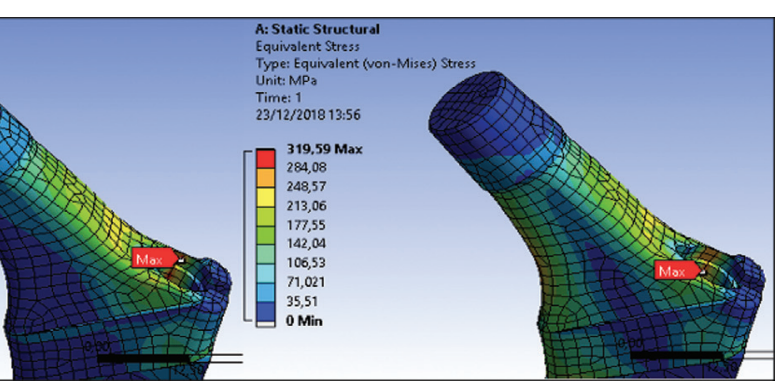

Tabela 4 Convergência da malha

\begin{tabular}{|l|l|l|l|l|}
\hline$\alpha^{\circ}$ & $\beta^{\circ}$ & $\begin{array}{l}\text { Tensão } \\
\text { equivalente } \\
(\mathrm{MPa})\end{array}$ & $\begin{array}{l}\text { Tensão } \\
\text { principal } \\
(\mathrm{MPa})\end{array}$ & $\begin{array}{l}\text { Deformação } \\
(\mathrm{mm} / \mathrm{mm})\end{array}$ \\
\hline 10 & 9 & 291,69 & 283,92 & 0,00142 \\
\hline 10 & 4 & 284,63 & 277,33 & 0,00139 \\
\hline 10 & 14 & 299,15 & 290,93 & 0,00146 \\
\hline 5 & 9 & 322,26 & 316,07 & 0,00158 \\
\hline 5 & 4 & 318,72 & 312,77 & 0,00157 \\
\hline 5 & 14 & 326,01 & 319,59 & 0,00160 \\
\hline 15 & 9 & 258,88 & 254,53 & 0,00125 \\
\hline 15 & 4 & 248,37 & 247,11 & 0,00120 \\
\hline 15 & 14 & 270,01 & 262,53 & 0,00130 \\
\hline
\end{tabular}


974 Análise da deformação na prótese femoral não cimentada do quadril Degasperi et al.

componente femoral em tensões elevadas e sua comparação com a análise pelo MEF.

\section{Conclusão}

A variabilidade de posicionamento da haste femoral protética de $+/-5^{\circ}$ no plano coronal e/ou a associação da angulação de $+/-5^{\circ}$ nos planos coronal e sagital interferiu de forma significativa na deformação do implante. Estudos clínicos com seguimento de longo prazo com próteses femorais de quadril não cimentadas devem ser realizados para verificar a influência do posicionamento excêntrico das hastes na sobrevida da artroplastia.

\section{Suporte financeiro}

Não houve suporte financeiro de fontes públicas, comerciais, ou sem fins lucrativos.

\section{Conflito de interesses}

Os autores declaram não haver conflito de interesses.

\section{Referências}

1 Evans JT, Evans JP, Walker RW, Blom AW, Whitehouse MR, Sayers A. How long does a hip replacement last? A systematic review and meta-analysis of case series and national registry reports with more than 15 years of follow-up. Lancet 2019;393(10172):647-654

2 Bergmann G, Deuretzbacher G, Heller M, et al. Hip contact forces and gait patterns from routine activities. J Biomech 2001;34(07): 859-871

3 Murray MP, Gore DR, Brewer BJ, Mollinger LA, Sepic SB. Joint function after total hip arthroplasty: a four-year follow-up of 72 cases with Charnley and Müller replacements. Clin Orthop Relat Res 1981;(157):119-124

4 ISO. 7206-4 Implants for surgery-Partial and total hip joint prostheses-Part 4: Determination of endurance properties and performance of stemmed femoral components. Vernier, Geneva
Switzerland 2010. Disponível em: https://www.iso.org/standard/ 69125.html

5 ISO. 7206-6 Implants for surgery-Partial and total hip joint prostheses-Part 6: Endurance properties testing and performance requirements of neck region of stemmed femoral components. Vernier, Geneva Switzerland 2013. Disponível em: https:// www.iso.org/standard/51186.html

6 Taylor M, Prendergast PJ. Four decades of finite element analysis of orthopaedic devices: where are we now and what are the opportunities? J Biomech 2015;48(05):767-778

7 Viceconti M, Toni A, Giunti A. Effects of some technological aspects on the fatigue strength of a cementless hip stem.J Biomed Mater Res 1995;29(07):875-881

8 Semenescu A, Radu-Ioniță F, Mateș IM, et al. Finite element analysis on a medical implant. Rom J Ophthalmol 2016;60(02): 116-119

9 ASTM F1713-08(2013) Standard Specification for Wrought Titanium-13Niobium-13Zirconium Alloy for Surgical Implant Applications (UNS R58130) American Society for Testing and Materials. 2013

10 Ravaglioli A, Krajewski C, Eds. Bioceramica e Corpo. Faenza, Italy: Springer Science + Business Media; 1984

11 Goel VK, Nyman E. Computational Modeling and Finite Element Analysis. Spine 2016;41(Suppl 7):S6-S7

12 Akrami M, Craig K, Dibaj M, Javadi AA, Benattayallah A. A threedimensional finite element analysis of the human hip. J Med Eng Technol 2018;42(07):546-552

13 Reimeringer M, Nuño N. The influence of contact ratio and its location on the primary stability of cementless total hip arthroplasty: A finite element analysis. J Biomech 2016;49(07): 1064-1070

14 Bitter T, Khan I, Marriott T, Lovelady E, Verdonschot N, Janssen D. Finite element wear prediction using adaptive meshing at the modular taper interface of hip implants. J Mech Behav Biomed Mater 2018;77:616-623

15 K N C, Zuber M, Bhat N S, Shenoy B S, R Kini C. Static structural analysis of different stem designs used in total hip arthroplasty using finite element method. Heliyon 2019;5(06):e01767

16 Delikanli YE, Kayacan MC. Design, manufacture, and fatigue analysis of lightweight hip implants. J Appl Biomater Funct Mater 2019;17(02):2280800019836830 